\title{
96
}

\section{Involving the school teacher in liberating the learner from traditional school culture}

\author{
John Turner \\ Presbyterian Ladies' College \\ Monash University \\ Victoria \\ Australia
}

\begin{abstract}
While computers generally have been available in schools since the early 1980s their contribution to constructivist learning within the classroom has been limited. Research centred around school teacher involvement in a postprimary school is shedding new light on using computers. The research focuses on the school mathematics culture within the school. Support materials which have emanated from the research, aid reflection on use of computers. The study suggests that the teacher can be a valued participant in constructivist change from within, even though the influence of some traditional school requirements remains a concern.
\end{abstract}

Main conference themes: teacher education

Educational areas: secondary education

Study topics: mathematics

Secondary keywords: culture, learning materials, logo, pedagogy, tools 


\section{CONSTRUCTIVISM}

Constructivism is an oft debated concept and it is not the purpose of this paper to discuss the merits of philosophical or psychological interpretations. Within the context of the 'constructivist classroom' where "students search for meaning, appreciate uncertainty and inquire responsibly" [1], constructivism is considered as a process where the learner is actively creating his or her knowledge. Computer constructivism refers to the use of computer technologies by the learner within this process.

In a similar vein the 'traditional school' and 'school mathematics' are phrases often used within computer constructivist research to signify negative connotations of formal schooling. It is only through understanding the responsibilities, opportunities and limitations of the contemporary school that realistic curriculum progress within such environments might be achieved. Rather than debating the relevance of the existence of formal schooling this paper considers a contemporary school whose teachers are well aware of and committed to constructivist learning as part of their curriculum responsibilities. From within this culture greater understanding is sought.

\section{INTRODUCTION}

Teachers in an independent postprimary (12-18 year old) girls' school in Melbourne, Victoria, on the basis of sufficient experience in using computers across the curriculum considered computer use in personally challenging environments for all students. However, they were not convinced that this would imply total replacement of noncomputer approaches. Despite the computer constructivist's view that the computer can enhance learning through being programmed by the learner or through acting as tutee [2] teachers did not see computer based learning as a core concern.

Teachers also saw computer education research, even when based in schools, as being too homogenous, ignoring community and curriculum requirements, and seeking answers predominantly from a computer viewpoint. Such research lend little, if any, practical support for school based curriculum improvement. Since 1980 considerable research has been done on advancing student centred learning within mathematics education in general [3], on school mathematics in Victoria [4] and on the 'constructivist classroom' [1]. Such research provided little if any substantive reason for the establishment of dominant computer constructivist environments within the school.

The teachers saw educational research as too often involving either the researcher controlling the environment or at least the stimuli and had little 
inclination or time to involve themselves. In exceptions such as the Logo Action Research Collaborative (LARC) [5] teachers appeared to be predisposed towards the software.

\section{Research Approach}

As an alternative approach to investigating computer use it was decided by the school's mathematics teachers to consider change from the bottom-up, from within the curriculum and subject to the active critical involvement of all the Mathematics teachers. It was resolved that:

- Change would only occur through the active participation of all teachers as decision makers.

- All mathematics teachers would be included so that the range of attitudes and approaches reflected all perspectives.

- Researcher support, while required, would adopt the role of 'supportive participant-observer' providing computer expertise and working in partnership with the teachers.

As an initial focus the Logo programming language was selected. Logo has been widely promoted as a model for constructivist learning and the "best example of the tutee mode" [6]. Although it had been used within the school's mathematics curriculum since the early 1980s the teachers felt that Logo may have outlived its usefulness. Through clarifying Logo's role the teachers hoped not only to resolve their concerns about its value for student learning, but also to find an approach for investigating other software types within the school. Similarities between Logo: "in the Logo environment .... children embark on an exploration about how they think" [7] and spreadsheeting: "a spreadsheet helps pupils explore, express and formalise their informal ideas" [8] indicated links worth pursuing.

\section{Research methodology}

The teachers worked through selfreflection and group decision making in line with the tradition in the Mathematics Department. Changes to the culture of the department generally came about through its leading members. The student voice and their expectations were important parts of teacher deliberations.

The support researcher's role was to work with the teachers, consider their input, suggest strategies based on the researcher's Logo experiences and skills, but ultimately to work to teacher direction. The researcher was a fellow teacher, although not an active teacher of mathematics. He worked with the teachers through research cycles which involved the following:

- Step 1: Teachers and researcher work together to identify changing needs. 
- Step 2: Researcher with teacher support generates stimuli to meet the needs.

- Step 3: The stimuli is introduced within the school's Mathematics culture.

- Step 4: Teachers with researcher support reflect on reactions to the stimuli.

In addition to observing all parts of the cycle the researcher interacted with the environment through questionnaires and interviews involving all mathematics teachers and school curriculum leaders. Observation included mathematics classes, both with and without computer use, and teacher interactions (formally and informally) outside the classroom. Student input was also gained through observation of their interaction with their teachers, questionnaires and consideration of the work done. Video and audio coverage provided avenues for deeper exploration as well as technical validation.

\section{Starting conditions}

After an initial period of observation and interaction a picture of the school mathematics culture and its use of Logo emerged. Personal development of students was the prime focus of each teacher's interest; a segmented topic curriculum, common testing and senior year curriculum and assessment requirements influenced student learning expectations and teacher decision making. Testing in conjunction with teacher experience and observation was the major tool used by teachers to gauge student progress.

Computing was primarily included in the mathematics curriculum so that students in their senior years could effectively use the computer to solve problems. Teachers generally wanted students to build up a toolkit which they could then effectively use to solve mathematical problems. Teachers also wanted each student's computer experiences linked to the curriculum topic at the time. For example, Logo turtle graphics was seen as relevant to studies in geometry.

Support material had been used with the Logo software. This had developed out of selections from books which 'taught' Logo to problem solving and investigative vignettes. Teachers felt that the material lacked mathematical credibility and too often failed to link together or with curriculum requirements.

The problems concerning computer use from structural, curriculum and philosophical viewpoints were discussed by the mathematics teachers and the researcher. Structural weaknesses were identified which pointed to disjointed, nondevelopmental student experiences. Insufficient time was available for a student to develop any worthwhile Logo toolkit. Blocked timetable use of computers gave little continuity to this development. And within Logo classrooms teachers were unsure of their capacity to achieve meaningful personal relationships between the student, the computer, the curriculum and themselves. Although content to use computers per se and being very 
productive in such use, students too often failed to comprehend the opportunities open to them. Logo use was heading towards use of the computer as 'experiences in isolation'. It was 'doing computers' and not much else. Teacher skill development was not progressing to the teachers' satisfaction, with some having progressed little beyond simple turtle graphics.

While the teachers wanted progress they found little research applicable within the school framework. Commercial publications provided limited support, generally treating Logo as a subject to be taught or as an open field for experimentation and did not address mathematics concerns.

In response Tasking Notes were develop which took into account teacher requirements while supporting Logo's constructivist philosophy. These notes encouraged student investigation and supported reflection while maintaining school mathematics curriculum credibility and promoting teacher involvement. Links were provided to parts of the school curriculum while covering the range of Logo mathematical concepts. The notes also addressed one common criticism of constructivism, that 'as a pedagogical framework it subordinates the curriculum to the interest of the child' [10]. The support material created learning 'microworlds' for both teacher and student.

\section{Refinement of stimulus}

Over a two-year period teacher and student use of this material was monitored. Important observations during this period included:

- Blocked timetable bookings involved long time gaps in which skills consequently diminished. This undermined continuity of advancement. Teachers were also adversely affected by the time gaps. Rotation of teachers each year also adversely affected continuity.

- Some teachers did not feel in control of the physical environment because of jammed printers and system malfunctions.

- Incoming (Year 7) students came from several feeder schools and brought with them a wide range of skills and attitudes.

- Some students were able to link their computer experiences with their mainstream topics, while others were content to work the tasks in a separate linear manner.

- Teachers remained unhappy with the gap between Logo and the language of school mathematics. They were also unsure of their capabilities to support individual student progress into the more advanced Logo concepts.

The Tasking Notes were reworked into a Tasking Workbook for each student. 


\section{Logo/Maths Workbook \\ Index}

How to Use this Workbook Debugging

Introduction to Using LogoWriter

Starting Logo Toolkit

A1: Block Letters
A2: Geometric Shapes
A3: Regular Polygons
A4: Circles
A5: Arcs
B1: Repeating Patterns (I)
B2: Repeating Patterns (II)
B3: Repeating Patterns (III)
B4: Star Patterns
B5: Further Star Patterns
C1: Movement and Music
C2: Words \& Stamps
C3: Multimedia Project
D1: Cartesian Placement
D2: Direction
D3: Cartesian Games

G1: Regular Polygon Variables

G2: Rectangle Variables

G3: Triangle Variables

G4: Trigonometry Problem Solving

H1: Variable Sub-procedures

H2: Variable Problem Solving

I1: Discussion Inputs

I2: Formulae

I3: Graphic Mathematical Explanations

I4: Output Tools

J1: Step-By-Step Guide to Recursion

J2: Order of Recursion

J3: Replacement Recursion

J4: Levels of Recursion

J5: Recursive Problem Solving

J6: Tail Recursion

J7: Randomised Recursion

Fig 1 Part of the Tasking Workbook index

The structure and content of the workbook addressed needs in various ways.

- Students were required to include working plans and completed work (procedures and graphics) in the workbook. This helped overcome time gaps and let the teacher see student thinking.

- Technical information as a reference/tutorial was included to ease demand on teachers.

- Toolkit support modules for each task were provided to enable students to acquire relevant Logo concepts and skills which the teachers may not possess.

- Students could start where they wanted.

- There was no preset structure of expectations within the workbook, although problems towards the end were of a senior year standard.

- Content included coverage of all Logo concepts to allow a problem solving 'tool-kit' to be built in conjunction with the mathematics content. 
- A 'two-tiered' tasking approach was developed where follow-up tasks were included to support reflective understanding through extension or lateral demands. This was necessary to minimize superficial responses to tasks.

Planning and solution space was provided to support student reflection and student/teacher communication.

\section{Toolkit: Tail Recursion}

Tail Recursion occurs when the procedure call is listed as the last instruction before END.

\section{Example Procedure}

TO PRINT.NUMBERS :num

IF :num $=0$ [STOP]

$\mathrm{PR}$ :num

PRINT.NUMBERS :num - 1

END

See how the procedure PRINT.NUMBERS is included in its own procedure as the last instruction before END.

Fig. 2 Part of the recursion toolkit module from section J6

\section{Task P4: Applying Logo Experiences to Solve Problems}

This problem was adapted from part of a 1992 VCE Common Assessment Task.

A float in the Moomba parade is a rectangle 12 metres long and 2.5 metres wide travelling at a constant speed of $u$ metres per second in a straight line along a road. A clown runs around the outside edge of the float at a speed of two metres per second continuously in a clockwise direction, always keeping within a negligible distance from the edge of the float. You may assume that the clown is at all times on the edge of the float.

Give sketches of the different types of paths which the clown traces out on the roadway for different values of $u$.

Fig. 3 'Float and clown': task from senior mathematics curriculum

Important issues in compiling the workbook included:

- Balancing support material with the amount of time allocated to using Logo. The workbook provided skill development and opportunity for exploration, but as a consequence asked greater time commitment from teachers and their students. 
- The linking of the support material and the curriculum was crucial. The workbook allowed each student to select starting position as well as task order, although some tasks required prior Logo skills and/or concepts.

- Each task could be approached either as a new learning experience, an alternative approach to learning a mathematical concept or as a problem solving task requiring the application of a previously acquired mathematical concept. In this way the workbook helped widen the topic centred curriculum by creating a multidimensional learning environment.

- The workbook used a more flexible student centred approach to content and problem choice. Teachers were therefore able to use the workbook as a facilitating support.

Task A2 Example

Create a Logo procedure that will draw at least two separate block letters of the alphabet to show a word, initials or an acronym.

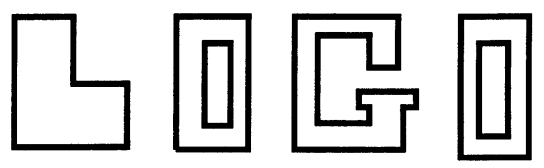

Include sub-procedures.

\section{Follow-Up Task}

Adapt your Logo procedure so that it will draw a mirror reflection of the letters. Copying and pasting your original procedure before adapting it is a good way to go.
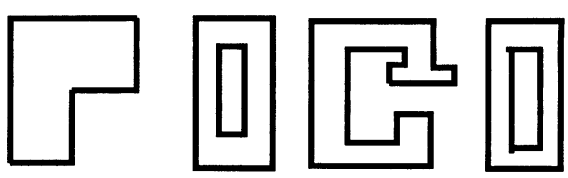

Fig. 4 Two-tiered task: reflection of a modular graphic program

\section{GENERAL OUTCOMES}

There is a range of issues emerging from the development and use of the workbook. Among the most significant are those related to the teacher's role and Logo confidence, including:

- the impact of limited resources; 
- teacher comfort with the technological environment;

- pressures in supporting different student starting points in skills and attitudes;

- the impact of learning philosophies on student and teacher attitudes and choices.

Many issues identified are crucial to achieving any computer objectives within the contemporary school. Teacher technical apprehensions need to be considered as part of teacher development as these impact on willingness to use computers in certain ways. If teachers are considered legitimate members of any change then they need to be made aware of the conditions which they work under.

The Tasking Workbook has provided enhanced support for using the computer as a problem solving tool. While examples of improved student learning due to constructivist opportunities have been forthcoming, highlighting individual examples would deny the structural limitations and the influence of assessment priorities which remain in contemporary school. While the study supports Papert's [9] contention regarding the incompatibility of the tutee potential of the computer with such structures, it has also lent support to the belief that working from within the structure still important constructivist advancement can be made. The workbook further remains an active and ongoing catalyst for teacher reflection and professional consideration.

The research process continues in that the models created for research and workbook structure are being used to consider other software packages across the curriculum.

\section{Task C2}

Select two comparable rectangular rooms (such as two classrooms or two rooms at home). Make an estimation of the difference in the area between the two rooms you selected.

Use a spreadsheet to calculate the area for each room and to compare the difference in sizes.

\section{Follow-Up Task}

Choose one of the rooms from task $\mathrm{C} 2$ and create a spreadsheet that can calculate the width for any given length so that the area remains the same as the room you have chosen. Use only whole metre lengths.

Graph the length against the width. (What can you conclude?)

Fig. 5 Spreadsheet example based on Logo workbook model 


\section{CONCLUSION}

Teachers are either facilitators of the learning environment or defenders of outmoded expectations. Teachers as leaders in change can produce positive progress in the pedagogical use of computers to improve student constructivist learning. Staff development requirements should take account of the impact of intermittent use of technology, the technical constraints of not always agreeable computer environments and the value of teachers as worthwhile contributors to productive change. In addition to the factors identified by Papert [9] school structure also hinders new more constructivist curricula. The research process and workbook model described have in part identified and addressed such factors.

\section{REFERENCES}

1. Brooks, J. and Brooks, M. (1993) In Search of Understanding: The Case for the Constructivist Classroom. Association for Supervision \& Curriculum Development, Alexandria, VA.

2. Taylor, R. (ed.) (1980) The Computer in the School: Tutor, Tool, Tutee. Teachers College Press, New York.

3. Davis, R., Maher, C. and Noddings, N. (eds.) Journal for Research in Mathematics Education-Monograph Number 4: Constructivist Views on the Teaching and Learning of Mathematics. National Council of Teachers of Mathematics, Virginia.

4. Clements, M., Grimmison, L and Ellerton, N. (1989) Colonialism and School Mathematics in Australia 1788-1988, in School Mathematics: the Challenge to Change. (eds Ellerton, N. and Clements, M.), Deakin University Press, Geelong, Victoria.

5. Watt, D. and Watt, M. (1993) New Paradigms in Classroom Research on Logo Learning. International Society for Technology in Education, Eugene, Oregon.

6. Squires, D. and McDougall, A. (1994) Choosing and Using Educational Software: A Teachers' Guide. Falmer Press, London 
7. Papert, S. (1980) Mindstorms: Children, Computers and Powerful Ideas. Harvester Press.

8. Sutherland, R. and Rojano, T. (1992) A Spreadsheet Approach to Solving Algebra Problems. (Unsourced)

9. Papert, S. (1993) The Children's Machine: Rethinking School in the Age of the Computer. Basic Books, New York. 